\title{
Electrochemiluminescence of dual excited states modulated with arginine modification of black phosphorus quantum dots
}

Huangxian Ju ( $\nabla$ hxju@nju.edu.cn )

Nanjing Universtiy https://orcid.org/0000-0002-6741-5302

Siqi Yu

Nanjing University

Yu Du

Nanjing University

Xianghong Niu

Nanjing University of Posts and Telecommunications https://orcid.org/0000-0001-6475-8839

Guangming Li

Nanjing University

Da Zhu

Nanjing University

Qian Yu

Nanjing University

Guizheng Zou

Shandong University, Jinan, China https://orcid.org/0000-0002-3295-3848

Article

Keywords:

Posted Date: February 10th, 2022

DOI: https://doi.org/10.21203/rs.3.rs-1309624/v1

License: (1) (1) This work is licensed under a Creative Commons Attribution 4.0 International License.

Read Full License

Version of Record: A version of this preprint was published at Nature Communications on November 26th, 2022. See the published version at https://doi.org/10.1038/s41467-022-35015-9. 


\section{Abstract}

The electrochemiluminescence $(E C L)$ is generally emitted via radiative transition of singlet or triplet excited state (S1 or T1). Herein, an ECL mechanism with the transitions of both S1 and T1 of black phosphorus quantum dots (BPQDs) was found for the first time, and an arginine modification strategy was proposed to passivate the surface oxidation defects of BPQDs, which could modulate the excited states for enhancing the ECL of BPQDs. The Arg modification led to greater spatial overlap of HOMO with LUMO and spectral shift of radiative transitions, and improved the stability of anion radical of BPQDs. The enhanced cathodic ECL was used to construct a sensitive method for conveniently evaluating the inhibiting efficiency of cyclo(RGDyK) to cell surface integrin by using RRGDS peptide modified BPQDs as signal tag. The dual excited states mediated ECL emitters provided a new paradigm for adjustable ECL generation and extended the application of ECL analysis.

\section{Full Text}

The electrochemiluminescence $(E C L)$ is generally emitted via radiative transition of singlet or triplet excited state $\left(S_{1}\right.$ or $\left.T_{1}\right)$. Herein, an ECL mechanism with the transitions of both $S_{1}$ and $T_{1}$ of black phosphorus quantum dots (BPQDs) was found for the first time, and an arginine modification strategy was proposed to passivate the surface oxidation defects of BPQDs, which could modulate the excited states for enhancing the ECL of BPQDs. The Arg modification led to greater spatial overlap of HOMO with LUMO and spectral shift of radiative transitions, and improved the stability of anion radical of BPQDs. The enhanced cathodic ECL was used to construct a sensitive method for conveniently evaluating the inhibiting efficiency of cyclo(RGDyK) to cell surface integrin by using RRGDS peptide modified BPQDs as signal tag. The dual excited states mediated ECL emitters provided a new paradigm for adjustable ECL generation and extended the application of ECL analysis.

Electrochemiluminescence (ECL) is a light-emitting process, in which the excited state species $\left(R^{*}\right)$ are generated via exergonic electron transfer and exchange between the electrogenerated intermediates, following the radiative transitions to the ground state $\left(\mathrm{S}_{0}\right)^{1}$. Generally, the produced $\mathrm{R}^{*}$ can be either the lowest excited singlet state $\left(S_{1}\right)$ species $\left({ }^{1} R^{*}\right)$ or the triplet state $\left(T_{1}\right)$ species $\left({ }^{3} R^{*}\right)$, depending on the difference of their relative energy ${ }^{2,3}$. According to the rules of spin statistics, the internal quantum efficiency of the luminophores emitting ECL is limited to $25 \%$ due to their ${ }^{1} R^{*}$-to- ${ }^{3} R^{*}$ ratio of $1: 3$ and the non-radiative transition from $\mathrm{T}_{1}$ to $\mathrm{S}_{0}{ }^{4}$, which limits the full utilization of the energy during the ECL process. In this work we found for the first time a possibility to emit the ECL via both $S_{1}$-to $S_{0}$ and $T_{1}$-to- $S_{0}$ transitions by using black phosphorus quantum dots (BPQDs) as the emitter.

BPQDs were firstly prepared in 2014 with bulk black phosphorus $(\mathrm{BP})^{5,6}$, which is a metal-free semiconductor and displays tunable bandgap varying from $0.3 \mathrm{eV}$ for bulk BP to $2.0 \mathrm{eV}$ for monolayer $\mathrm{BP}^{7}$, and have been extensively applied in photothermal therapy, electrocatalysis and flexible devices $5,6,8-$

${ }^{10}$. However, their optical and electrical performances are greatly limited by the oxidation defects of 
nanostructure surface due to the extremely easy degradability under ambient conditions ${ }^{11-13}$. Great efforts have been made to stabilize BPQDs by either preventing the occurrence of oxidation process via encapsulation with polyethylene glycol, fluorine and poly(lactic-co-glycolic acid) , $^{7,14}$, or passivating the existing oxidation defects with ethanol ${ }^{15}$. Meanwhile, arginine ( $\mathrm{R}$ or $\mathrm{Arg}$ ) contained peptides and poly- ${ }^{-}$ lysine have also been used to modify BP nanosheets via electrostatic and/or hydrophobic interaction for the preparation of delivery carrier and the immobilization of protein, respectively ${ }^{16,17}$. Inspired by these interaction mechanisms, this work designed a strategy to passivate the oxidation defects of BPQDs with Arg for enhancing the ECL performance.

Here, the ECL emission of BPQDs can be attributed to the radiative transitions of both ${ }^{1} R^{*}$ and ${ }^{3} R^{*}$ to $S_{0}$ species. Although the radiative $\mathrm{T}_{1}$-to- $\mathrm{S}_{0}$ transition has been observed in the ECL emission of some organic small molecules with phosphorescence $(\mathrm{PL})$ properties, such as benzophenone, platinum-based organometallics and tris(1-phenyl isoqsuinoline-C2, N)iridium (III) ${ }^{18-20}$, the ECL mechanism mediated by dual excited states has not been reported yet. More interestingly, the cathodic and anodic ECL emission upon Arg modification of BPQDs could increase dramatically by 25 and 2 folds, respectively, which resulted from the change of the highest occupied molecular orbital (HOMO) from the surface oxidation defects to the central zone of R-BPQDs. This change caused greater spatial overlap of the HOMO with the lowest unoccupied molecular orbital (LUMO), as proved by time-dependent density functional theory (TDDFT) calculations, and the adjustable transition routes from $S_{1}$ and $T_{1}$ to $S_{0}$. Thus Arg modification could be used to efficiently modulate the excited states for enhancing the ECL emission. Due to the improved stability of anion radical of BPQDs, the cathodic ECL showed greater improvement of performance than anodic ECL. More importantly, the Arg functionalization can improve the solubility and modifiability of BPQDs for extending the bioanalytical applications. As an example, an ECL system was proposed for conveniently evaluating the integrin inhibitor by using RRGDS-BPQDs as signal tag to recognize cell surface integrin. This work demonstrated a novel ECL mechanism and an exciting avenue to modulate the excited states for enhancing the ECL emission of luminophores.

\section{Results}

Synthesis and characterization of BPQDs and R-BPQDs. BPQDs were prepared with a solvothermal method $^{5,6}$, and then functionalized with Arg to obtain R-BPQDs (Fig. 1a). From the transmission electron microscopic (TEM) images, their average lateral sizes were measured to be about $4 \mathrm{~nm}$ (Figs. 1b,c). The lattice fringes of $0.23 \mathrm{~nm}$ could be ascribed to the (041) plane of the BP crystal (Inset in Fig. 1b) ${ }^{21}$. The measured heights of 1.1, 1.8 and $2.4 \mathrm{~nm}$ (Supplementary Fig. 1) corresponded to BPQDs with about 2-4 layers, respectively ${ }^{5,6}$. The Fourier transform infrared (FTIR) spectra showed the shifts of $\mathrm{P}-\mathrm{O}$ and $\mathrm{N}-\mathrm{H}$ bending peaks from $1070 \mathrm{~cm}^{-1}$ of BPQDs and $1721 \mathrm{~cm}^{-1}$ of Arg to 1089 and $1705 \mathrm{~cm}^{-1}$ of R-BPQDs, respectively (Fig. 1d) ${ }^{22,23}$, indicating the electrostatic and hydrogen bond interactions between the electron-withdrawing guanidine group in $\mathrm{Arg}$ and $\mathrm{P}_{\mathrm{x}} \mathrm{O}_{\mathrm{y}}$ moiety ${ }^{24,25}$. The Arg modification also led to a slight red-shift of three prominent Raman peaks of BPQDs (Fig. 1e), identified as one out-of-plane phonon 
mode $\left(A_{g}^{1}\right)$ at $360.1 \mathrm{~cm}^{-1}$ and two in-plane modes $\left(B^{2}{ }_{g}\right.$ and $\left.A^{2}{ }_{g}\right)$ at 435.5 and $462.7 \mathrm{~cm}^{-1} 5,6$, which manifested a decrease of the Raman scattering energy due to the interaction between guanidine group and $\mathrm{P}_{\mathrm{x}} \mathrm{O}_{\mathrm{y}}$ moiety ${ }^{26}$. The R-BPQDs retained the characteristic $\mathrm{P} 2 \mathrm{p}_{3 / 2}$ and $\mathrm{P} 2 \mathrm{p}_{1 / 2} \mathrm{X}$-ray photoelectron spectroscopic (XPS) peaks of BP at 128.6 and $129.3 \mathrm{eV}$ (Fig. $1 \mathrm{f}$ and Supplementary Figs. 2a,b), while $B P Q D s$ did not show these peaks, demonstrating that the introduction of Arg endowed BPQDs with better stability. The shifts of $\mathrm{P}-\mathrm{O}$ and $\mathrm{P}=\mathrm{O}$ XPS peaks from 132.6 and $133.6 \mathrm{eV}$ of $\mathrm{BP}$ to 134.8 and $135.9 \mathrm{eV}$ of R-BPQDs could be attributed to the decrease of the outer valence electron density of $P$ atom in the presence of guanidine group (Fig. $1 \mathrm{f}$ and Supplementary Fig. 2b), which did not obviously change the N1s XPS peak due to the delocalized positive charge distribution over the guanidine group (Supplementary Figs. 2c,d), but increased the binding energy of $\mathrm{O}$ atom for $\mathrm{P}-\mathrm{O}$ and $\mathrm{P}=\mathrm{O}$ bonds due to the decreased outer valence electron density (Supplementary Figs. 2e-h). In a word, the presence of Arg on the PxOy moiety of BPQDs exerted a passivation effect against the oxidation defects of BPQDs (Fig. 1g).

Photophysical and electrochemical properties of BPQDs and R-BPQDs. The cathodic process of BPQDs/GCE showed an ambiguous reduction peak of BPQDs at around $-1.22 \mathrm{~V}$, which shifted to $-1.15 \mathrm{~V}$ and became more distinct after introducing the electron-withdrawing guanidine group by Arg modification (Fig. 2a). In the presence of $\mathrm{K}_{2} \mathrm{~S}_{2} \mathrm{O}_{8}, \mathrm{R}-\mathrm{BPQDs} / \mathrm{GCE}$ showed a cathodic ECL emission at $-1.20 \mathrm{~V}$, which was 25 folds stronger than that of BPQDs/GCE (Fig. 2b). The ECL emission followed a general $\mathrm{K}_{2} \mathrm{~S}_{2} \mathrm{O}_{8}$ mediated co-reactant ECL mechanism containing the reduction of $\mathrm{K}_{2} \mathrm{~S}_{2} \mathrm{O}_{8}$ and $\mathrm{BPQDs}$ or R-BPQDs to produce the excited state BPQDs ${ }^{*}$ or R-BPQDs ${ }^{*}$ (Inset in Fig. 2b). The reduction peak of $\mathrm{K}_{2} \mathrm{~S}_{2} \mathrm{O}_{8}$ at bare GCE occurred at $-0.95 \mathrm{~V}$, and almost disappeared at BPQDs/GCE due to the greatly increased electron transfer impedance $(R e)$ (Supplementary Fig. 3). The much lower Re than BPQDs/GCE led to obvious reduction peak of $\mathrm{K}_{2} \mathrm{~S}_{2} \mathrm{O}_{8}$ at R-BPQDs/GCE, which overlapped with the reduction peak of R-BPQDs (Fig. 2a). The consistence of onset potentials for electrochemical reduction and cathodic ECL emission of R-BPQDs indicated that the electrogeneration of R-BPQDs ${ }^{*-}$ was necessary for the formation of excited state R-BPQDs ${ }^{\star 27}$. Interestingly, the ECL depended on both Arg concentration and $\mathrm{pH}$ for preparation of RBPQDs (Supplementary Fig. 4), implying that the amount of Arg assembled on BPQDs affected the ECL performance, though Arg did not participate in the ECL process (Supplementary Fig. 5).

The FL and PL emission of BPQDs centering at 505 and $580 \mathrm{~nm}$ (Fig. 2c) originated from the oxidation defects associated $S_{1}$ and $T_{1}$, respectively ${ }^{8,15}$, which was demonstrated by the photoluminescence decay spectrum ${ }^{15}$ (PDS) (Supplementary Fig. 6). The PDS of both BPQDs and R-BPQDs showed biexponential functions with lifetime components of 3.08 and $12.27 \mu$ s and 1.82 and $13.14 \mu \mathrm{s}$, respectively(Supplementary Table 1), indicating the presence of two decay channels assigned to the two excited states for FL and PL emissions, respectively ${ }^{28}$. R-BPQDs displayed $45-\mathrm{nm}$ and 20 -nm blue shift and the increased FL and PL emission intensity, respectively. The hypochromic shift and intensity enhancement were related to the change of the two excited states due to the passivation of oxidation defects by Arg. Excitingly, the cathodic ECL spectra of both BPQDs and R-BPQDs displayed two emission 
peaks at 500 and $580 \mathrm{~nm}$ for BPQDs, and 460 and $570 \mathrm{~nm}$ for R-BPQDs (Fig. 2d). Compared with the FL and PL spectra of BPQDs and R-BPQDs (Fig. 2c), the ECL emission peaks at 500 and $460 \mathrm{~nm}$ could be attributed to the transition from $S_{1}$, while the peaks at 580 and $570 \mathrm{~nm}$ originated from $T_{1}$-to- $S_{0}$ transition. Thus it could be concluded that ECL emissions of BPQDs and R-BPQDs contained two radiative transitions from both $S_{1}$ and $T_{1}$, as shown in Fig. 2e. After Arg modification, the hypochromic shifts of both $\mathrm{S}_{1}$-to- $\mathrm{S}_{0}$ and $\mathrm{T}_{1}$-to- $\mathrm{S}_{0}$ transitions were observed in the ECL spectra, which was consistent with larger experimental band gap of R-BPQDs than BPQDs (Supplementary Fig. 7). However, their lifetimes did not obviously change at temperatures from 170 to $310 \mathrm{~K}$ (Supplementary Fig. 8 and Table 2), so the possibility of thermally activated delayed fluorescence process could be excluded ${ }^{4,29}$, further indicating the existence of two radiative transitions from both $S_{1}$ and $T_{1}$ for ECL emission.

According to the electrostatic and hydrogen bond interactions between Arg and oxidation defects of BPQDs, TD-DFT computation was implemented to rationalize above conclusion. The existence of oxidation defects resulted in the localized HOMO of BPQDs at the defect sites (Fig. 2f), which hindered the charge transfer as a trap state, and thus weakened the FL, PL and ECL intensity. The Arg modification passivated the surface oxidation defects, accordingly leading to the delocalization of HOMO of R-BPQDs to the central zone (Fig. 2f), and thus the change of the electron transition channel, which significantly improved the emission oscillator strength and the charge transfer capability (Supplementary Fig. 3). Besides, R-BPQDs exhibited the strongest ECL emission and the most positive reduction potential among the BPQDs modified with 20 kinds of amino acids (Supplementary Table 3), demonstrating the significance of electron-withdrawing guanidine group, which stabilized the adjacent R-BPQDs ${ }^{*-}$ anion radical after electrochemically injecting electron into LUMO of R-BPQDs ${ }^{30,31}$, and thus facilitated the cathodic ECL emission.

The BPQDs/GCE showed two anodic peaks at +0.85 and $+1.45 \mathrm{~V}$ (Fig. 3a), which were attributed to the electrochemical oxidation of surface groups such as phosphite and hypophosphoric groups ${ }^{25,32}$. These peaks negatively shifted to +0.42 and $+0.86 \mathrm{~V}$ after Arg modification (Fig. 3a) due to the much lower $R e$ (Supplementary Fig. 3), which decreased the oxidation overpotentials. Although the oxidation of Arg could be observed at bare GCE at $+1.62 \mathrm{~V}$ (Supplementary Fig. 9), it did not occur at R-BPQDs/GCE in the applied potential range due to the relative higher $R e$. Considering the low oxidation potential of R-BPQDs, $\mathrm{N}_{2} \mathrm{H}_{4} \bullet \mathrm{H}_{2} \mathrm{O}$ that can be electrochemically oxidized to produce $\mathrm{N}_{2} \mathrm{H}_{3} \cdot$ and $\mathrm{N}_{2} \mathrm{H}_{2}$ around $+0.1 \mathrm{~V}$ was used as co-reactant to study the anodic ECL of BPQDs and R-BPQD s ${ }^{33,34}$. At bare GCE, the oxidation of $\mathrm{N}_{2} \mathrm{H}_{4} \bullet \mathrm{H}_{2} \mathrm{O}$ occurred near $+0.10 \mathrm{~V}$, which showed a peak at $+0.55 \mathrm{~V}$ and a severe tailed anodic curve due to the further oxidation of $\mathrm{N}_{2} \mathrm{H}_{3}$ and $\mathrm{N}_{2} \mathrm{H}_{2}$ at higher potentials (Fig. 3b). Obviously, the anodic curve of $\mathrm{N}_{2} \mathrm{H}_{4} \bullet \mathrm{H}_{2} \mathrm{O}$ positively shifted due to the increased $R e$, and covered the oxidation peaks of BPQDs at both BPQDs/GCE and R-BPQDs/GCE. Furthermore, the hole-injected BPQDs $\left(B P Q D s^{\circ+}\right.$ ) could oxidize the reducing $\mathrm{N}_{2} \mathrm{H}_{3}{ }^{*}$, $\mathrm{N}_{2} \mathrm{H}_{2}$ and $\mathrm{N}_{2} \mathrm{H}_{4}$ to form the excited state species BPQDs ${ }^{*}$ or R-BPQDs ${ }^{*}$ for ECL emission (Fig. $3 \mathrm{C}$ ). The anodic ECL peak potential and intensity of R-BPQDs were $0.15 \mathrm{~V}$ lower and 2 times higher than those of BPQDs, which could be attributed to the better charge transfer capability, the greater spatial overlap 
between HOMO and LUMO, and the better stability under ambient conditions (Supplementary Fig. 10) after Arg modification. Similar to the cathodic ECL process, the anodic ECL spectra of BPQDs and RBPQDs also displayed two emission peaks associating $S_{1}$ and $T_{1}$ transitions, along with the hypochromic shifts (Supplementary Fig. 11). Thus the co-reactant ECL mechanisms of R-BPQDs at the cathode and the anode could be illustrated in Fig. 3d.

$\mathrm{ECL}$ transient technology was further used to examine the stability of radical intermediates in two ECL processes of R-BPQDs. The ion annihilation ECL intensity at $+1.60 \mathrm{~V}$ was stronger than that at $-1.40 \mathrm{~V}$ in the absence of coreactant (Fig. 3e), indicating that the anion radical R-BPQDs ${ }^{-{ }^{-}}$was more stable than cation radical R-BPQDs ${ }^{*+35}$. Thus Arg stabilized the anion radical of BPQDs under ambient conditions, and thus led to the greater enhancement of cathodic ECL intensity than the anodic process.

Evaluation of integrin inhibitor with RRGDS-BPQDs. To implement the application of R-BPQDs $/ \mathrm{K}_{2} \mathrm{~S}_{2} \mathrm{O}_{8}$ ECL system, this work designed an ECL method to evaluate the inhibiting efficiency of integrin inhibitor, cyclo(RGDyK) ${ }^{36}$, by using Arg containing peptide RRGDS to modify BPQDs (RRGDS-BPQDs). The RRGDSBPQDs functionalized carboxylated multi-wall carbon nanotubes (MWNTs) were coated on GCE to act as both the recognition unit and signal tag ${ }^{37}$. Compared to Arg-free peptide GGGDS, the presence of RRGDS could greatly enhance the ECL intensity (Fig. 4a), verifying the vital importance of Arg for improving the $\mathrm{ECL}$ emission of BPQDs. Upon the specific recognition of $\mathrm{aV} / \beta 3$ integrin on $\mathrm{A} 549$ cell membrane with RRGDS on GCE, the Re increased greatly (Supplementary Fig. 12), and thus the ECL intensity decreased obviously (Supplementary Fig. 13). In contrary, MCF-7 cells with low abundance of surface $a V / \beta 3$ integrin showed little decrease. Under optimal conditions (Supplementary Figs. 14,15), the IC50 of cyclo(RGDyK) for $1^{\prime} 10^{6}$ A549 cells $\mathrm{mL}^{-1}$ was obtained to be $12.0 \mathrm{nM}$ from the ECL response plot of cyclo(RGDyK) treated A549 cells (Fig. 4b), which was comparable to $20 \mathrm{nM}$ for immobilized $\mathrm{aV} / \beta 3$ integrin $^{36}$ and 29.3 nM for B16-F10 cells ${ }^{38}$. Compared to general MTT method, this method was sensitive, simple and convenient. Moreover, this method could be extended for the evaluation of other inhibitors or the detection of cell surface groups by changing the Arg-containing peptide, showing the excellent practicability of the designed ECL modulating strategy.

\section{Discussion}

The BPQDs showed the lattice fringes ascribed to the (041) plane of the BP crystal with 2-4 layers 5,6 , and could be conveniently modified with Arg via the electrostatic and hydrogen bond interactions (Fig. 1d). The interactions led to decreased Raman scattering energy ${ }^{26}$ and outer valence electron density of $P$ atom (Fig. 1f). The presence of Arg on BPQDs could passivate the oxidation defects of BPQDs (Fig. 1g), and thus endowed BPQDs with better stability, solubility and modifiability for extending the bioanalysis application.

The ECL emission of BPQDs showed a novel mechanism containing two radiative transitions from both $\mathrm{S} 1$ and $\mathrm{T} 1$ to $\mathrm{S}_{0}$ (Fig. 2e), which were demonstrated by the ECL spectra with two emission peaks (Fig. 2d) 
as observed from the FL and PL emission of BPQDs (Fig. 2c). Thus a dual excited states mediated ECL emitter was found for the first time. The modification of Arg led to the hypochromic shifts of both $\mathrm{S}_{1}$-to$S_{0}$ and $T_{1}$-to- $S_{0}$ transitions due to its passivation against the surface oxidation defects (Fig. $2 d$ ) to produce the delocalization of HOMO of R-BPQDs to the central zone, which changed the electron transition channel and was demonstrated by TD-DFT computation (Fig. 2f). By comparing the ECL change upon amino acid modification (Supplementary Table 3), it was concluded that the ECL enhancement of R-BPQDs was attributed to the presence of electron-withdrawing guanidine group, which could stabilize the adjacent R-BPQDs-- anion radical after electrochemically injecting electron into LUMO of R-BPQDs.

The Arg modification could be used to modulate the dual excited states mediated ECL emission due to the change of charge transfer capability and the spatial overlap between HOMO and LUMO, which led to enhanced ECL emission of BPQDs. Moreover, the enhancement of cathodic ECL emission was much greater than anodic ECL emission. This appearance was ascribed to the more stable anion radical R-

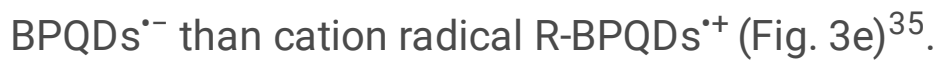

To demonstrate the application of R-BPQDs in ECL bioanalysis, the Arg attached on BPQDs was used to conjugate RGDS, a peptide specific to integrin ${ }^{36}$. By coating RRGDS-BPQDs on MWNTs modified GCE, the integrin-rich cells could be bound to the electrode surface via the recognition of RGDS to integrin ${ }^{39}$, which led to a sensitive ECL method for the evaluation of integrin inhibitor. The obtained inhibiting efficiency to integrin on A549 cells demonstrated the practicability of the ECL of BPQDs and the modulating strategy.

In summary, both $\mathrm{S}_{1}$-to- $\mathrm{S}_{0}$ and $\mathrm{T}_{1}$-to- $\mathrm{S}_{0}$ radiative transitions have been found in both cathodic and anodic ECL emission of BPQDs. Arg modification efficiently passivates the oxidation defects of BPQDs and changes the HOMO from surface defects to the central zone, thus leading to hypochromic shifts and intensity enhancement of ECL emission. The introduction of Arg changes the electron transition channel, and endows BPQDs with better stability, stronger charge transfer capability and greater spatial overlap between HOMO and LUMO. The presence of electron-withdrawing guanidine group greatly stabilizes the anion radical intermediates, and thus leads to greater enhancement of the cathodic ECL intensity. The proposed modulating strategy can be conveniently applied in biosensing by using different Argcontaining recognition units to modify BPQDs, which has been demonstrated by using RRGDS-BPQDs to evaluate the inhibiting efficiency of cyclo(RGDyK) to cell surface integrin. The discovery of dual excitedstates mediated ECL mechanism and the modulation strategy via Arg modification open a new avenue to decipher more ECL systems for broadening the ECL applications of nanoemitters.

\section{Methods}

Materials and reagents. N-hydroxysuccinimide (NHS), L-arginine (Arg), L-histidine (His), 1-(3(dimethylamino)propyl)-3-ethylcarbodiimide hydrochloride (EDC) and hydrazine hydrate solution $\left(\mathrm{N}_{2} \mathrm{H}_{4} \cdot \mathrm{H}_{2} \mathrm{O}, 64-65 \%\right)$ were purchased from Sigma-Aldrich (Merck, USA). 1-Methyl-2-pyrrolidinone (NMP) 
was purchased from J\&K Chemical Technology Co., Ltd. (Beijing, China). L-Lysine monohydrochloride (Lys), L-isoleucine (lle), L-valine (Val), L-asparagine monohydrate (Asp), L-phenylalanine (Phe), L-leucine (Leu), L-threonine (Thr), L-glutamine (GIn), L-alanine (Ala), L-serine (Ser), L-glutamic acid (Glu), potassium hexacyanoferrate( ) $\left(\mathrm{K}_{3}\left[\mathrm{Fe}(\mathrm{CN})_{6}\right]\right)$, potassium hexacyanoferrate( ) trihydrate $\left(\mathrm{K}_{4}\left[\mathrm{Fe}(\mathrm{CN})_{6} \cdot 3 \mathrm{H}_{2} \mathrm{O}\right]\right)$ and potassium peroxydisulfate $\left(\mathrm{K}_{2} \mathrm{~S}_{2} \mathrm{O}_{8}\right)$ were purchased from Hushi Co., Ltd. (Shanghai, China). L-Aspartic acid (Asp), L-glycine (Gly), L-proline (Pro), L-Cysteine (Cys), L-methionine (Met), L-tryptophan (Try) and Ltyrosine (Tyr) were obtained from Ryon Biological Technology Co., Ltd. (Shanghai, China). Bulk back phosphorus (BP) and carboxylated multi-walled carbon nanotubes (MWNTs) (20-30 nm diameter, 0.5-2 $\mu \mathrm{m}$ length) were obtained from Jiangsu XFNANO Materials Tech Co., Ltd. (Nanjing, China). 2-(Nmorpholino)ethanesulfonic acid (MES) buffer (0.1 M, pH 6.0) was purchased from Coolaber Technology Co., Ltd. (Beijing, China). Peptides, including RRGDS and GGGDS, were obtained from Sangon Biotech Co., Ltd. (Shanghai, China). The inhibitor of $\mathrm{aV} / \beta 3$ integrin, cyclo(RGDyK), was purchased from topscience Co., Ltd. (Shanghai, China). Fetal bovine serum (FBS) was obtained from Thermo Fisher Scientific Inc. (USA). A549 cells, MCF-7 cells, trypsin and cell culture media (RPMI-1640) were supplied by KeyGen Biotech Co., Ltd. (Nanjing, China). All chemicals were of analytical grade. Phosphate buffer solution (PBS, $0.1 \mathrm{M}, \mathrm{pH}$ 7.4) was prepared by mixing stock solutions of $\mathrm{NaH}_{2} \mathrm{PO}_{4}$ and $\mathrm{Na}_{2} \mathrm{HPO}_{4}$. $\mathrm{ECL}$ measurements were conducted in $0.1 \mathrm{M} \mathrm{pH} 7.4 \mathrm{PBS}$ containing $0.1 \mathrm{M} \mathrm{KNO}_{3}$ or $0.1 \mathrm{M} \mathrm{KCl}$ as the electrolyte. All aqueous solutions were prepared using ultrapure water ( $\geq 18 \mathrm{M} \Omega \cdot \mathrm{cm}$, Milli-Q, Millipore).

Preparation and functionalization of BPQDs. The BPQDs were prepared by top-down solvothermal treatment according to the literature with some modification ${ }^{5}$. Briefly, $15 \mathrm{mg}$ of bulk BP crystals was firstly grounded into powder by a mortar and pestle in the glovebox, which was then dispersed in $15 \mathrm{~mL}$ of anhydrous NMP to heat for $12 \mathrm{~h}$ at $140{ }^{\circ} \mathrm{C}$ under Ar atmosphere. The resulting dark brown dispersion was centrifuged at $8000 \mathrm{rpm}$ for $20 \mathrm{~min}$ to remove the oversized particles. The supernatant was collected and further centrifuged at $18000 \mathrm{rpm}$ for $40 \mathrm{~min}$. The resultant BPQDs were redispersed in $15 \mathrm{~mL}$ of anhydrous NMP and kept in refrigerator at $4{ }^{\circ} \mathrm{C}$ for further use. To prepare amino acid- or peptidefunctionalized BPQDs, $1.0 \mathrm{~mL}$ of BPQDs dispersion was centrifuged at $21000 \mathrm{rpm}$ for $40 \mathrm{~min}$. The resultant BPQDs were redispersed in $1.0 \mathrm{~mL}$ of amino acid or peptide solution and sonicated in an icebath for $30 \mathrm{~min}$. Afterward, the mixture was shaken overnight at room temperature away from light. The resultant solution was centrifuged at $21000 \mathrm{rpm}$ for $40 \mathrm{~min}$ and washed three times with water. Finally, the functionalized BPQDs, such as RRGDS-BPQDs and GGGDS-BPQDs et al., were redispersed in deoxygenated water and kept at $4{ }^{\circ} \mathrm{C}$ in the dark.

Cell culture and inhibitor treatment. A549 and MCF-7 cells were cultured in RPMI-1640 media supplemented with $10 \% \mathrm{FBS}$ and $100 \mu \mathrm{g} \mathrm{mL}^{-1}$ penicillin-streptomycin at $37^{\circ} \mathrm{C}$ in a humidified atmosphere containing $5 \% \mathrm{CO}_{2}$ to maintain a density between $5 \times 10^{5}$ and $2 \times 10^{6}$ cells $\mathrm{mL}^{-1}$. At the logarithmic growth phase, the cells were trypsinized and washed twice with sterile $0.01 \mathrm{M} \mathrm{pH} 7.4$ PBS by centrifugation at $1000 \mathrm{~g}$ for $10 \mathrm{~min}$, which were then resuspended in $0.01 \mathrm{M} \mathrm{pH} 7.4$ PBS containing 1.0 $\mathrm{mM} \mathrm{Ca}^{2+}$ and $1.0 \mathrm{mM} \mathrm{Mg}^{2+}$ to obtain a homogeneous cell suspension. The inhibitor treatment of the 
cells was performed by incubating $200 \mu \mathrm{L}$ of $1 \times 10^{6} \mathrm{~A} 549$ cells $\mathrm{mL}^{-1}$ for $20 \mathrm{~min}$ with different concentrations of $\mathrm{aV} / \beta 3$ integrin inhibitor, cyclo(RGDyK), on a rotary shaker in the dark at $37^{\circ} \mathrm{C}$. After washing with PBS for three times, the treated cells were redispersed in $200 \mu \mathrm{L}$ of $0.01 \mathrm{M} \mathrm{pH} 7.4 \mathrm{PBS}$ for ECL evaluation of inhibiting efficiency.

QDs modified GCEs. GCEs were firstly polished with 0.3 and $0.05 \mu \mathrm{m}$ alumina slurry sequentially and sonicated in ethanol and distilled water for $3 \mathrm{~min}$. The cleaned electrodes were dried with a steam of high-purity nitrogen gas. $10 \mu \mathrm{L}$ of BPQDs, amino acid-functionalized BPQDs or peptide-functionalized $B P Q D$ s dispersion was then individually cast onto the GCEs and dried under ambient condition to get the QDs modified GCEs.

Fabrication of ECL sensor for IC50 determination. $10 \mu \mathrm{L}$ dispersion of $5 \mathrm{mg} \mathrm{mL}^{-1}$ carboxylated MWNTs was dropped on the pretreated GCE and dried in a desiccator to get MWNTs/GCE. $20 \mu \mathrm{L}$ of $0.1 \mathrm{M}$ MES buffer containing $10 \mathrm{mM} \mathrm{EDC}$ and $5 \mathrm{mM}$ NHS was then dropped onto MWNTs/GCE to incubate for $1 \mathrm{~h}$ at room temperature. After the activated MWNTs/GCE was thoroughly rinsed with $0.01 \mathrm{M} \mathrm{pH} 7.4 \mathrm{PBS}, 20 \mu \mathrm{L}$ of RRGDS-BPQDs was immediately dropped on its surface to incubate for $2 \mathrm{~h}$ at room temperature. The resulting RRGDS-BPQDs/MWNTs/GCE was rinsed with $0.01 \mathrm{M} \mathrm{pH} \mathrm{7.4} \mathrm{PBS,} \mathrm{and} \mathrm{then} \mathrm{was} \mathrm{dropped} \mathrm{with} 20$ $\mu \mathrm{L}$ of $1 \times 10^{6}$ inhibitor-pretreated $\mathrm{A} 549$ cells $\mathrm{mL}^{-1}$ to incubate at $37^{\circ} \mathrm{C}$ for $20 \mathrm{~min}$. After carefully rinsing with $0.01 \mathrm{M} \mathrm{pH}$ 7.4 PBS, the obtained A549/RRGDS-BPQDs/MWNTs/GCE was used for subsequent assay. As control, MCF-7/RRGDS-BPQDs/MWNTs/GCE was prepared with a similar procedure.

ECL spectra. After $10 \mu \mathrm{L}$ of BPQDs or R-BPQDs was dropped on GCE and dried, the cathodic and anodic $\mathrm{ECL}$ spectra of the modified electrodes were recorded in $0.1 \mathrm{M} \mathrm{pH} 7.4$ PBS containing $0.1 \mathrm{M} \mathrm{K}_{2} \mathrm{~S}_{2} \mathrm{O}_{8}$ or 25 $m M N_{2} \mathrm{H}_{4} \cdot \mathrm{H}_{2} \mathrm{O}$, respectively, with an optical filter from 380 to $700 \mathrm{~nm}$ in front of the PMT.

Calculation method. All calculations were carried out using the Amsterdam Density Functional program package (ADF) ${ }^{40}$. The time-dependent density functional theory (TD-DFT) and DFT calculations were performed by applying the Perdew-Burke-Ernzerhof $(\mathrm{PBE})^{41}$ exchange-correlation functional with the triple-zata plus polarization (TZP) basis set ${ }^{42}$. The ground state configuration, $\mathrm{S}_{0}$, of the oxidized BPQDs and R-BPQDs, was first optimized by DFT calculation. To simulate the emission, the configurations of the lowest singlet state, $S_{1}$, and the lowest triplet state, $T_{1}$, were relaxed using TD-DFT starting from the ground state configuration. We computed the vertical electronic transitions from the excited state relaxed configurations $S_{1}$ and $T_{1}$ to the ground-state, yielding the fluorescence and phosphorescence, respectively. The Van der Waals interaction was taken into account by the semi-empirical D3 method proposed by Grimme et $\mathrm{al}^{43}$. All optimizations were done without any symmetry constraint.

\section{Data availability}

The data that support the findings of this study are available within the article and supplementary information files, or from the corresponding author upon request. 
The authors did not use any previously unreported custom computer code or algorithm.

\section{Declarations}

\section{Acknowledgements}

We gratefully acknowledge the National Natural Science Foundation of China $(21890741,21827812$, 21635005).

\section{Author contributions}

S.Q. Yu, Y. Du and H.X. Ju proposed the idea and designed the experiments. S.Q. Yu, Y. Du, X.H. Niu and H.X. Ju wrote the manuscript. S.Q. Yu carried out the synthesis and electrochemical experiments. X.H. Niu carried out the structure simulation and theoretical calculations. G.M. Li performed the photoluminescence experiments. D. Zhu and Q. Yu helped in electrochemistry measurements. G.Z. Zou and H.X. Ju supervised and coordinated all investigators for this project. All authors discussed the results and commented on the manuscript.

\section{Competing interests}

The authors declare no competing interests.

\section{Additional information}

Supplementary information The online version contains supplementary material available at https://doi.org/10.1038/....

Correspondence and requests for materials should be addressed to Huangxian Ju.

Peer review information Nature Communications thanks the anonymous reviewers for their contribution to the peer review of this work. Peer reviewer reports are available.

Reprints and permission information is available online at http://npg.nature.com/reprints

Publisher's note Springer Nature remains neutral with regard to jurisdictional claims in published maps and institutional affiliations.

\section{References}

1. Zhao, Y., Yu, J., Xu, G., Sojic, N. \& Loget, G. Photoinduced electrochemiluminescence at silicon electrodes in water. J. Am. Chem. Soc. 141, 13013-13016 (2019). 
2. Rizzo, F. et al. From blue to green: fine-tuning of photoluminescence and electrochemiluminescence in bifunctional organic dyes. J. Am. Chem. Soc. 139, 2060-2069 (2017).

3. Irkham. et al. Electrogenerated chemiluminescence by in situ production of coreactant hydrogen peroxide in carbonate aqueous solution at a boron-doped diamond electrode. J. Am. Chem. Soc. 142, 1518-1525 (2020).

4. Ishimatsu, R. et al. Electrogenerated chemiluminescence of donor-acceptor molecules with thermally activated delayed fluorescence. Angew. Chem., Int. Ed. 53, 6993-6996 (2014).

5. Zhang, X. et al. Black phosphorus quantum dots. Angew. Chem., Int. Ed. 54, 3653-3657 (2015).

6. Sun, Z. et al. Ultrasmall black phosphorus quantum dots: synthesis and use as photothermal agents. Angew. Chem., Int. Ed. 54, 11526-11530 (2015).

7. Gusmão, R., Sofer, Z. \& Pumera, M. Black phosphorus rediscovered: from bulk material to monolayers. Angew. Chem., Int. Ed. 56, 8052-8072 (2017).

8. Liu, J., Liu, T., Du, P., Zhang, L. \& Lei, J. Metal-organic framework (MOF) hybrid as a tandem catalyst for enhanced therapy against hypoxic tumor cells. Angew. Chem., Int. Ed. 58, 7808-7812 (2019).

9. Shao, J. et al. Biodegradable black phosphorus-based nanospheres for in vivo photothermal cancer therapy. Nat. Commun. 7, 12967 (2016).

10. Xu, Z. et al. Exceptional catalytic effects of black phosphorus quantum dots in shuttling-free lithium sulfur batteries. Nat. Commun. 9, 4164 (2018).

11. Zhou, Q., Chen, Q., Tong, Y. \& Wang, J. Light-induced ambient degradation of few-layer black phosphorus: mechanism and protection. Angew. Chem., Int. Ed. 55, 11437-11441 (2016).

12. Abellán, G. et al. Fundamental insights into the degradation and stabilization of thin layer black phosphorus. J. Am. Chem. Soc. 139, 10432-10440 (2017).

13. Favron, A. et al. Photooxidation and quantum confinement effects in exfoliated black phosphorus. Nat. Mater. 14, 826-832 (2015).

14. Tang, X. et al. Fluorination-enhanced ambient stability and electronic tolerance of black phosphorus quantum dots. Adv. Sci. 5, 1800420 (2018).

15. Long, L. et al. Highly fluorescent and stable black phosphorus quantum dots in water. Small 14, 1803132 (2018).

16. Zhou, W., Cui, H., Ying, L. \& Yu, X.-F. Enhanced cytosolic delivery and release of CRISPR/Cas9 by black phosphorus nanosheets for genome editing. Angew. Chem., Int. Ed. 57, 10268-10272 (2018).

17. Zhao, Y. et al. Synthesis of a poly-L-lysine/black phosphorus hybrid for biosensors. Anal. Chem. 90, 3149-3155 (2018).

18. Park, S. M. \& Bard, A. J. The production of benzophenone phosphorescence in fluid solution by radical ion reaction. Chem. Phys. Lett. 38, 257-262 (1976).

19. Mak, C. H. et al. Thermally activated delayed phosphorescence and interchromophore exciton coupling in a platinum-based organometallic emitter. Adv. Opt. Mater. 8, 2001023 (2020). 
20. Guo, W., Ding, H., Zhou, P., Wang, Y. \& Su, B. Electrochemiluminescence waveguide in single crystalline molecular wires. Angew. Chem., Int. Ed. 59, 6745-6749 (2020).

21. Brown, A. \& Rundqvist, S. Refinement of the crystal structure of black phosphorus. Acta Crystallogr. 19, 684 (1965).

22. Zhu, X. et al. Stabilizing black phosphorus nanosheets via edge-selective bonding of sacrificial $\mathrm{C}_{60}$ molecules. Nat. Commun. 9, 4177 (2018).

23. Yang, S. et al. Azide passivation of black phosphorus nanosheets: covalent functionalization affords ambient stability enhancement. Angew. Chem., Int. Ed. 58, 1479-1483 (2019).

24. Chavali, S. S., Cavender, C. E., Mathews, D. H. \& Wedekind, J. E. Arginine forks are a widespread motif to recognize phosphate backbones and guanine nucleobases in the RNA major groove. J. Am. Chem. Soc. 142, 19835-19839 (2020).

25. Zhang, T. et al. Degradation chemistry and stabilization of exfoliated few-layer black phosphorus in water. J. Am. Chem. Soc. 140, 7561-7567 (2018).

26. Qiu, M. et al. Novel concept of the smart NIR-light-controlled drug release of black phosphorus nanostructure for cancer therapy. Proc. Natl. Acad. Sci. U. S. A. 115, 501-506 (2018).

27. Tan, X., Zhang, B. \& Zou, G. Electrochemistry and electrochemiluminescence of organometal halide perovskite nanocrystals in aqueous medium. J. Am. Chem. Soc. 139, 8772-8776 (2017).

28. Jiang, K., Wang, Y., Cai, C. \& Lin, H. Conversion of carbon dots from fluorescence to ultralong roomtemperature phosphorescence by heating for security applications. Adv. Mater. 30, 1800783 (2018).

29. Rajamalli, P. et al. New molecular design concurrently providing superior pure blue, thermally activated delayed fluorescence and optical out-coupling efficiencies. J. Am. Chem. Soc. 139, 1094810951 (2017).

30. Nepomnyashchii, A. B., Cho, S., Rossky, P. J. \& Bard, A. J. Dependence of electrochemical and electrogenerated chemiluminescence properties on the structure of BODIPY dyes. Unusually large separation between sequential electron transfers. J. Am. Chem. Soc. 132, 17550-17559 (2010).

31. Chiesa, M., Giamello, E. \& Che, M. EPR characterization and reactivity of surface-localized inorganic radicals and radical ions. Chem. Rev. 110, 1320-1347 (2010).

32. Gusmão, R., Sofer, Z. \& Pumera, M. Functional protection of exfoliated black phosphorus by noncovalent modification with anthraquinone. ACS Nano 12, 5666-5673 (2018).

33. Zheng, X., Zhang, Z., Guo, Z. \& Wang, Q. Flow-injection electro-generated chemiluminescence detection of hydrazine based on its in-situ electrochemical modification at a pre-anodized platinum electrode. Analyst 127, 1375-1379 (2002).

34. Li, Z., Wu, S., Zhang, B., Fu, L. \& Zou, G. Promising mercaptobenzoic acid-bridged charge transfer for electrochemiluminescence from CulnS $2 @ Z n S$ nanocrystals via internal $\mathrm{Cu}^{+} / \mathrm{Cu}^{2+}$ Couple cycling. J. Phys. Chem. Lett. 10, 5408-5413 (2019).

35. Ding, Z. et al. Electrochemistry and electrogenerated chemiluminescence from silicon nanocrystal quantum dots. Science 296, 1293-1297 (2002). 
36. Haubner, R. et al. Glycosylated RGD-containing peptides: Tracer for tumor targeting and angiogenesis imaging with improved biokinetics. J. Nuclear Med. 42, 326-336 (2001).

37. Cheng, W., Ding, L., Lei, J., Ding, S. \& Ju, H. Effective cell capture with tetrapeptide-functionalized carbon nanotubes and dual signal amplification for cytosensing and evaluation of cell surface carbohydrate. Anal. Chem. 80, 3867-3872 (2008).

38. Yin, J. et al. Cyclic RGDyK conjugation facilitates intracellular drug delivery of polymeric micelles to integrinoverexpressing tumor cells and neovasculature. J. Drug Targeting 19, 25-36 (2011).

39. Cheng, W., Ding, L., Ding, S., Yin, Y. \& Ju, H. A simple electrochemical cytosensor array for dynamic analysis of carcinoma cell surface glycans. Angew. Chem. Int. Ed. 48, 6465-6468 (2009).

40. ADF SCM, Theoretical Chemistry; Vrije Universiteit: Amsterdam, The Netherlands. http://www.scm.com.

41. Perdew, J. P., Burke, K. \& Ernzerhof, M. Generalized gradient approximation made simple. Phys. Rev. Lett. 77, 3865-3868 (1996).

42. Van Lenthe, E. \& Baerends, E. J. Optimized slater-type basis sets for the elements 1-118. J. Comput. Chem. 24, 1142-1156 (2003).

43. Grimme, S. Semiempirical GGA-type density functional constructed with a long-range dispersion correction. J. Comput. Chem. 27, 1787-1799 (2006).

\section{Figures}




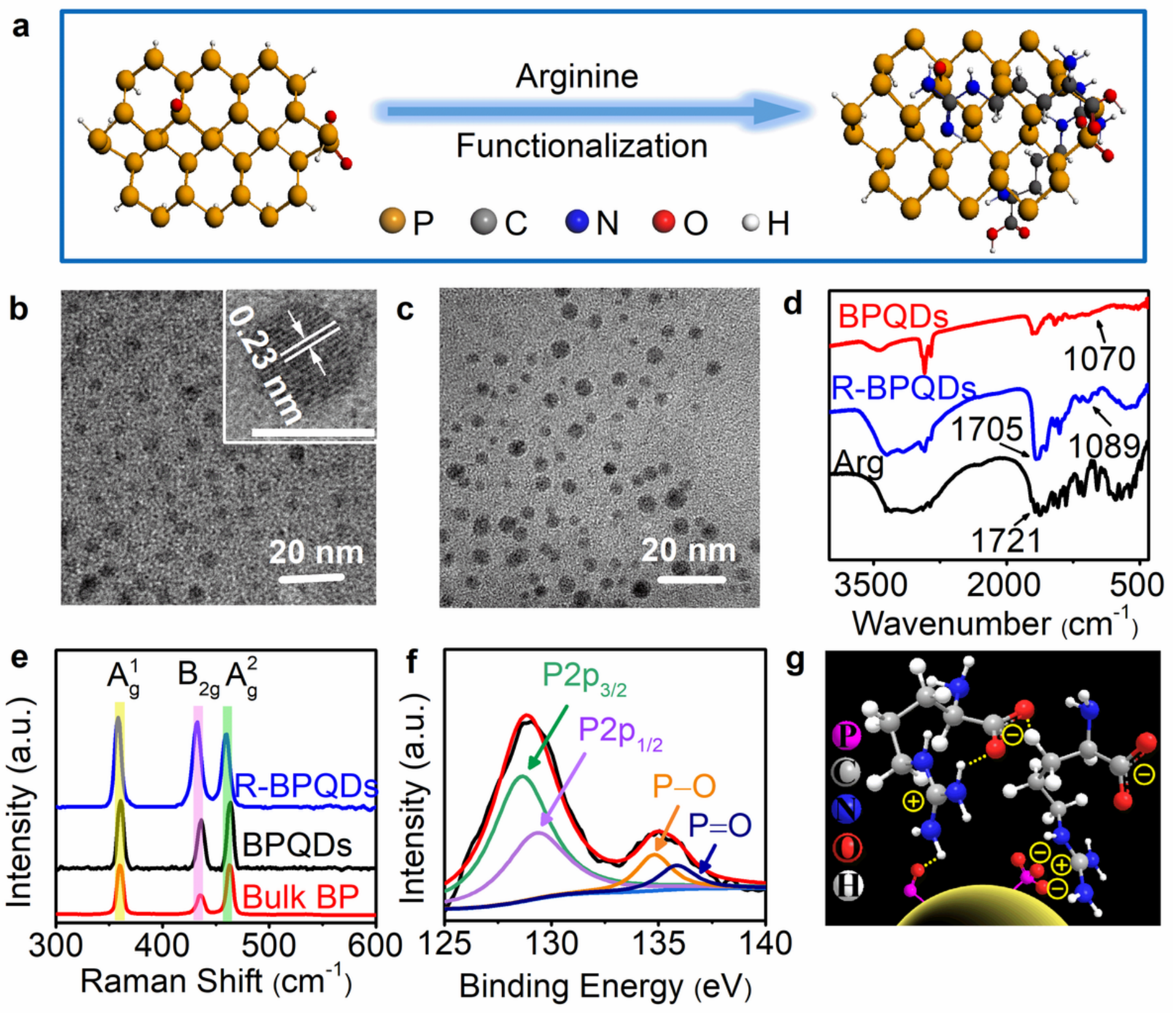

Figure 1

Synthesis and characterization of BPQDs and R-BPQDs. a Structures of BPQDs and R-BPQDs. TEM images of $\mathbf{b} B P Q D$ s and $\mathbf{c}$ R-BPQDs. Inset in $\mathbf{b}$ : HRTEM image of BPQDs with scale bar of $5 \mathrm{~nm}$. $\mathbf{d}$ FTIR spectra of Arg, R-BPQDs and BPQDs. e Raman spectra of bulk BP, BPQDs and R-BPQDs. $f$ Experimental (black) and fitting (red) P2p XPS spectra of R-BPQDs. g Schematic profile of interaction between BPQDs and Arg. 


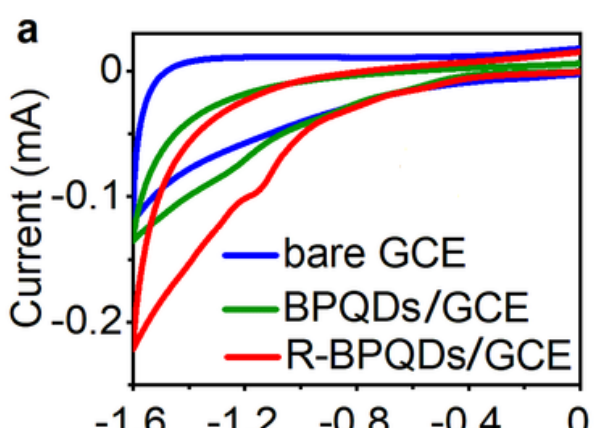

$\begin{array}{lllll}-1.6 & -1.2 & -0.8 & -0.4 & 0\end{array}$

Potential (V vs. Ag/AgCl)

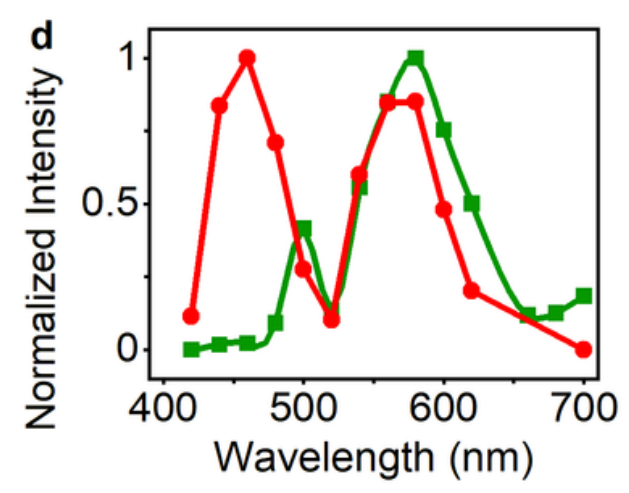

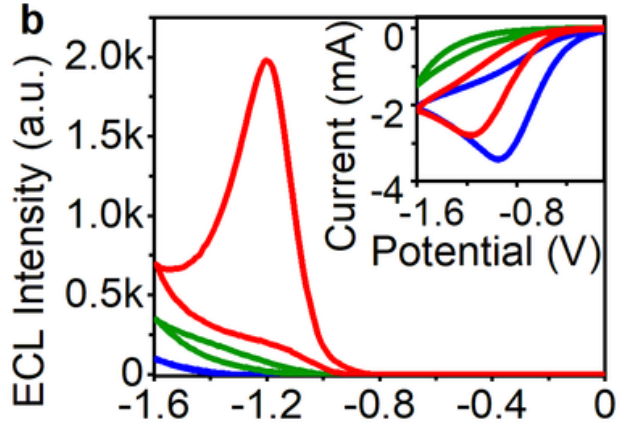

Potential (V vs. Ag/AgCl)

e

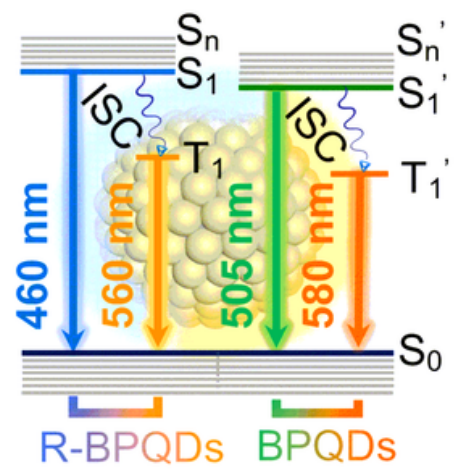

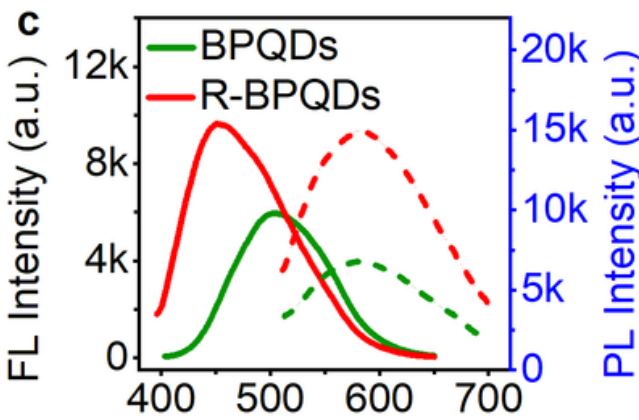

Wavelength $(\mathrm{nm})$

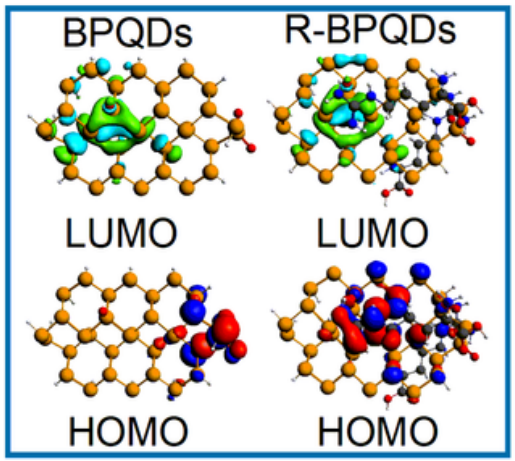

Figure 2

Cathodic ECL and photophysical properties of BPQDs and R-BPQDs. a CVs of GCEs in $0.1 \mathrm{M} \mathrm{PBS.} \mathbf{b}$ ECL curves of GCEs in $0.1 \mathrm{M} \mathrm{pH}$ 7.4 PBS containing $0.1 \mathrm{M} \mathrm{K}_{2} \mathrm{~S}_{2} \mathrm{O}_{8}$. PMT = $600 \mathrm{~V}$. Inset: CVs for b. c FL (solid lines, $\lambda_{\mathrm{ex}}=390 \mathrm{~nm}$ ) and PL (dashed lines, $\lambda_{\mathrm{ex}}=500 \mathrm{~nm}$ ) spectra of BPQDs and R-BPQDs. $\mathbf{d}$ Normalized cathodic ECL spectra of BPQDs/GCE and R-BPQDs/GCE. e Schematic illustration of ECL transitions of $B P Q D$ s and R-BPQDs. $f$ Isosurfaces of HOMO and LUMO wavefunctions for BPQDs and R-BPQDs. 

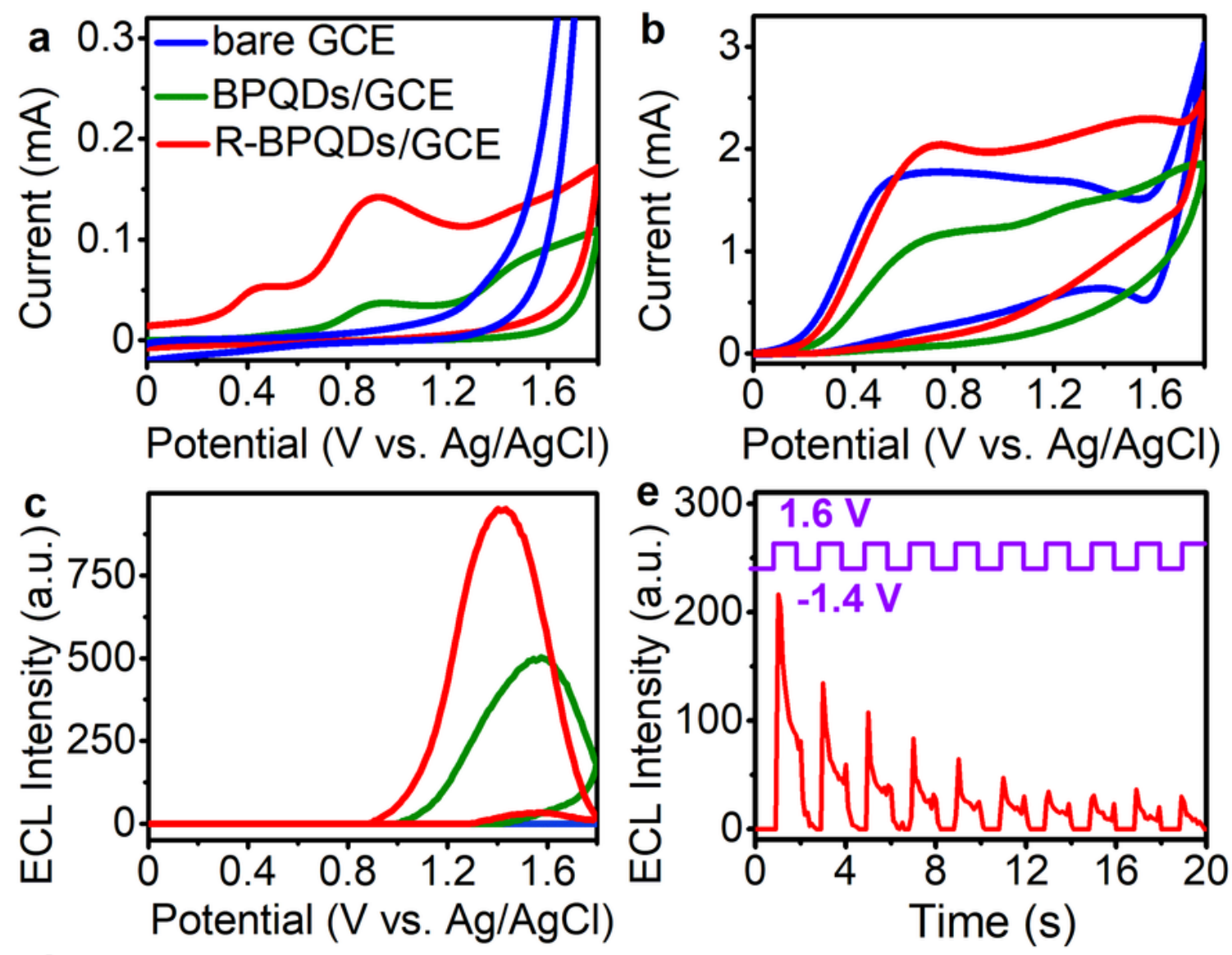

d

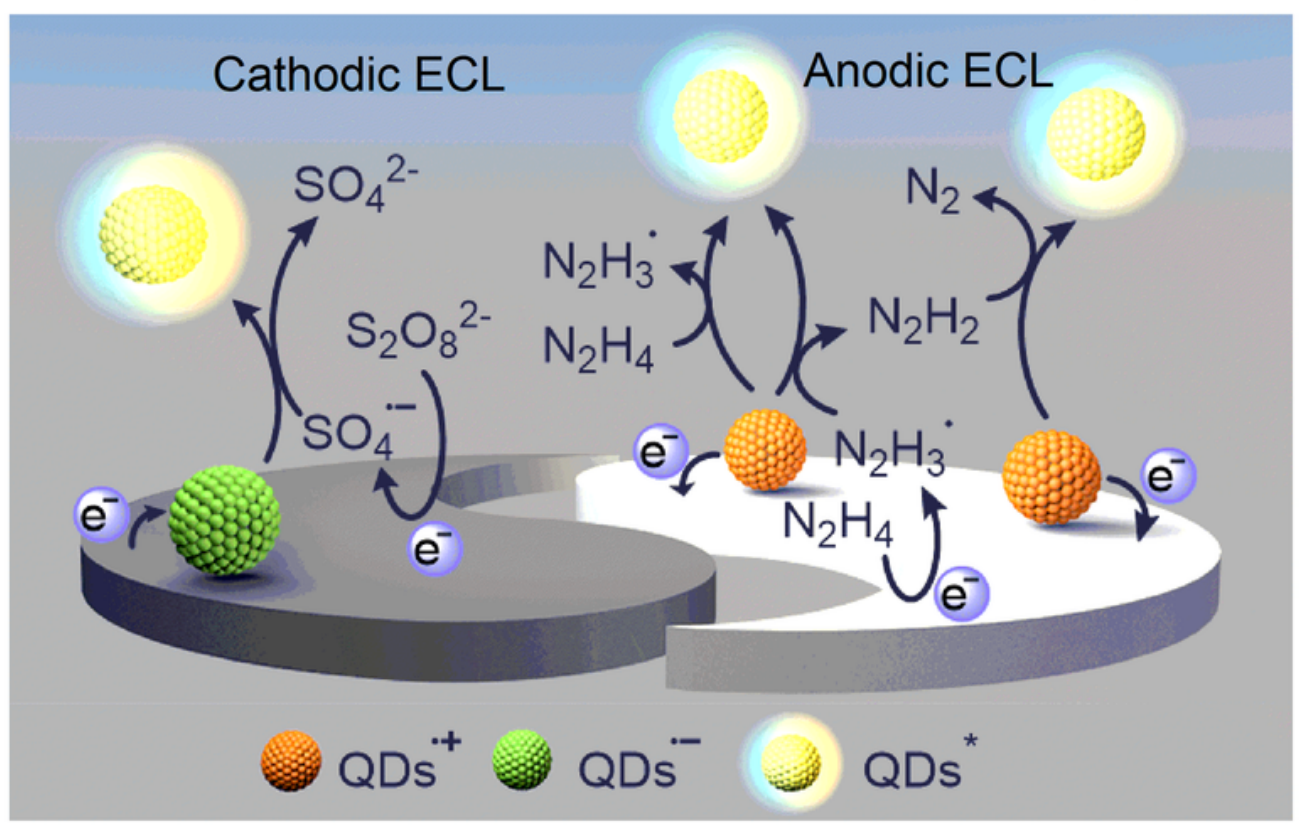

Figure 3

Anodic ECL, transient ECL and ECL mechanisms. $\mathbf{a}, \mathbf{b}$ CV and $\mathbf{c}$ anodic ECL curves of GCE, BPQDs/GCE and R-BPQDs/GCE in $0.1 \mathrm{M} \mathrm{pH} 7.4 \mathrm{PBS}$ in $\mathbf{a}$ absence and $\mathbf{b}$, $\mathbf{c}$ presence of $25 \mathrm{mM} \mathrm{N}_{2} \mathrm{H}_{4} \bullet \mathrm{H}_{2} \mathrm{O}$. PMT $=600$ V. $\mathbf{d}$ Cathodic and anodic ECL mechanisms of R-BPQDs/GCE in the presence of $\mathrm{K}_{2} \mathrm{~S}_{2} \mathrm{O}_{8}$ and $\mathrm{N}_{2} \mathrm{H}_{4} \cdot \mathrm{H}_{2} \mathrm{O}$, respectively. e ECL transient of R-BPQDs/GCE in $0.1 \mathrm{M}$ PBS by stepping potential from $-1.4 \mathrm{~V}$ to $+1.6 \mathrm{~V}$ at $1 \mathrm{~Hz}$. 

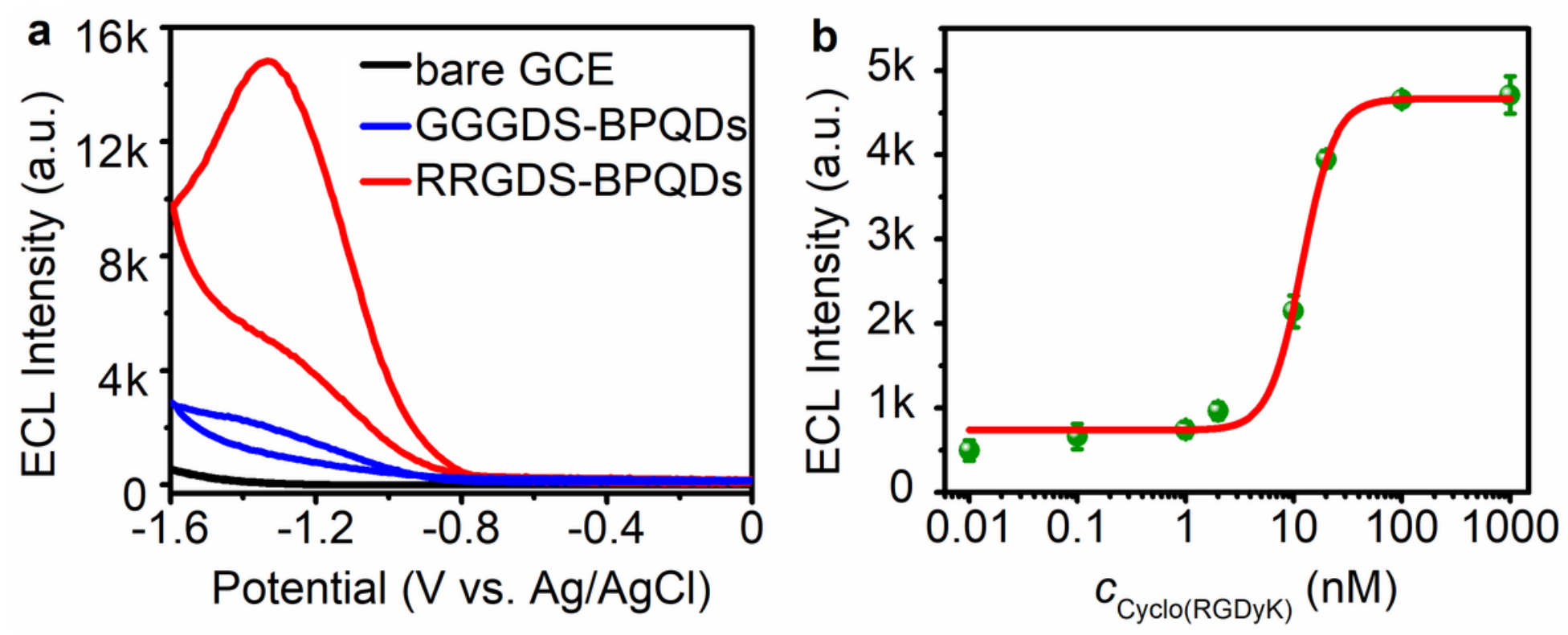

Figure 4

Evaluation of integrin inhibitor. a ECL curves of GCE, GGGDS-BPQDs/GCE and RRGDS-BPQDs/GCE in 0.1 $\mathrm{M}$ PBS containing $0.1 \mathrm{M} \mathrm{K}_{2} \mathrm{~S}_{2} \mathrm{O}_{8}$. PMT = $800 \mathrm{~V}$. b ECL responses of RRGDS-BPQDs/MWNTs/GCE to $1{ }^{\prime}$ $10^{6} \mathrm{~A} 549$ cells $\mathrm{mL}^{-1}$ pretreated with $0.01,0.1,1,2,10,20,100$ and $1000 \mathrm{nM}$ cyclo(RGDyK).

\section{Supplementary Files}

This is a list of supplementary files associated with this preprint. Click to download.

- SIXXtoXXNatCommun.docX

- NCOMMS2203659RS.pdf 